\title{
On hydrodynamic shear turbulence in Keplerian disks: Via transient growth to bypass transition
}

\author{
G. D. Chagelishvili ${ }^{1}$, J.-P. Zahn ${ }^{2}$, A. G. Tevzadze ${ }^{1}$, and J. G. Lominadze ${ }^{1}$ \\ ${ }^{1}$ Center for Plasma Astrophysics, Abastumani Astrophysical Observatory, Kazbegi 2a, 380060 Tbilisi, Georgia \\ e-mail: aleko@tevza.org \\ 2 LUTH, Observatoire de Paris, 92190 Meudon, France \\ e-mail: jean-paul .zahn@obspm.fr
}

Received 11 December 2002 / Accepted 6 February 2003

\begin{abstract}
This paper deals with the problem of hydrodynamic shear turbulence in non-magnetized Keplerian disks. Several papers have appeared recently on the subject, on possible linear instabilities which may be due to the presence of a stable stratification, or caused by deviations from cylindrical rotation. Here we wish to draw attention to another route to hydrodynamic turbulence, which seems to be little known by the astrophysical community, but which has been intensively discussed among fluid dynamicists during the past decade. In this so-called bypass concept for the onset of turbulence, perturbations undergo transient growth and if they have initially a finite amplitude they may reach an amplitude that is sufficiently large to allow positive feedback through nonlinear interactions. This transient growth is linear in nature, and thus it differs in principle from the well-known nonlinear instability. We describe the type of perturbations that according to this process are the most likely to lead to turbulence, namely non-axisymmetric vortex mode perturbations in the two dimensional limit. We show that the apparently inhibiting action of the Coriolis force on the dynamics of such vortical perturbations is substantially diminished due to the pressure perturbations, contrary to current opinion. We stress the similarity of the turbulent processes in Keplerian disks and in Cartesian flows and conclude that the prevalent skepticism of the astrophysical community about the occurrence of hydrodynamic shear turbulence in such disks is not founded.
\end{abstract}

Key words. accretion, accretion disks - hydrodynamics - instabilities - turbulence

\section{Introduction}

The emergence of X-ray astronomy exposed the importance of accretion phenomena in many astrophysical systems, such as binary stars, quasars and active galactic nuclei. The possible mechanisms governing accretion have been schematically understood during the very first years of investigations (Shakura \& Sunyaev 1973; Pringle 1981): the inward transport of matter and the outward transport of angular momentum in accretion disks was ascribed to a turbulent (anomalous) viscosity. The consequent development in understanding the physics of this phenomenon and the causes of that turbulence has been irregular and has taken considerable time. Substantial progress has been achieved in the nineties with the discovery of a linear instability in magnetized disks (Balbus \& Hawley 1991, 1992, 1998; Hawley \& Balbus 1991, 1992; Hawley et al. 1995; Stone et al. 1996). In contrast, the solution of the accretion problem in the non-magnetized case has not yet reached sufficient maturity. Moreover, the very occurrence of turbulence in non-magnetized disks has been questioned by Balbus et al. (1996) and Balbus \& Hawley (1998). The reason for this situation is that cylindrical flows with Keplerian profile belong to

Send offprint requests to: J.-P. Zahn, e-mail: jean-paul.zahn@obspm.fr the class of smooth shear flows, i.e. which present no inflection point; it is well known that these flows are spectrally stable, although they may become turbulent in the laboratory. The explanation of this behavior remains one of the fundamental problems of fluid mechanics. However the situation has changed in recent years, with the recognition that the so-called non-modal approach provides an adequate, and even an optimal mathematical formalism to describe the dynamics of perturbations in plane-parallel shear flows. This led to the emergence of the bypass concept for the onset of turbulence in spectrally stable flows. This concept has triggered much interest among fluid dynamicists, but it had little impact so far on the astrophysical community. For this reason we feel it necessary to present the salient results of that novel approach in a journal which is read by astrophysicists, to demonstrate its relevance for the dynamics of Keplerian disks, and to draw attention to the bibliography on the subject.

Our approach parallels that of Longaretti (2002), who analyzed the existing experimental and numerical results, and who also insisted, as we shall do here, on the similarity of subcritical shear turbulence in plane and rotating flows.

Our paper is organized as follows. In Sect. 2 we outline the fundamental problems of fluid mechanics bearing on the description of plane-parallel smooth shear flows. We present 
the recent developments in shear flow analysis made by the hydrodynamic community, and the novel bypass concept for the onset of turbulence in shear flows. In Sect. 3 we analyze the quadratic forms of the dynamical equations and discuss the essential role of pressure perturbations, which counteract the effect of the Coriolis force in 2D flows. In Sect. 4 we describe the transient growth of two dimensional perturbations. This will allow us to stress the similarity between the behavior of Keplerian and Cartesian flows, and the negligible role of the Coriolis force in the 2D perturbation kinematics and energetics. Finally we conclude in Sect. 5 that the bypass concept provides a plausible scenario leading to turbulence in astrophysical disks. In the Appendix we present a simple sketch of the bypass scenario.

\section{From spectral decay to transient growth and bypass transition}

\subsection{On the onset of turbulence in spectrally stable shear flows}

From the theoretical viewpoint there are flows that are spectrally stable at all Reynolds numbers (e.g. plane Couette or pipe Hagen-Poiseuille flows), while some others become spectrally unstable at high enough Reynolds numbers (e.g. plane Poiseuille or Blasius flows). In the latter case the flow is characterized by the critical Reynolds number that is the marginal value of this parameter above which the spectral instability occurs. In this sense the critical Reynolds number of the spectrally stable flow is infinity.

Here we shall consider smooth shear flows (without inflection point in the velocity profile), like in Keplerian disks. These are linearly stable according to classical fluid mechanics. As shown by Rayleigh (1880), the existence of an inflection point (more precisely of a vorticity extremum, as pointed out by Fjørtøft 1950) in the equilibrium velocity profile is a necessary condition for the occurrence of a linear (spectral) instability in hydrodynamic flows. Thus smooth shear flows (flows without a vorticity extremum) are "relaxed" in this context: they are spectrally stable, meaning that exponentially growing solutions are absent. However, it is well known from laboratory experiments and from numerical simulations that finite amplitude perturbations may cause a transition from laminar to turbulent state at moderate, less then critical Reynolds number.

This has led to the development of the concept of nonlinear instability in hydrodynamics (cf. Bayly 1986; Bayly et al. 1988; Herbert 1988; Orszag \& Kells 1980; Orszag \& Patera 1980, 1983). Until about ten years ago, the predominant view of this laminar-turbulent transition was centered around the slow linear amplification of exponentially growing perturbations (the familiar T-S waves), which modify the flow profile and thereby allow a secondary instability, further nonlinearity and finally a breakdown to turbulent flow. According to this concept of nonlinear instability, the perturbations (and the turbulent state itself) are energetically sustained by nonlinear processes. This concept of nonlinear instability has been borrowed by astrophysicists, who still use it to explain turbulent processes in smooth astrophysical flows, where no spectrally unstable solution is known, and in particular in Keplerian disks flows (see Balbus \& Hawley 1998). However, there are subcritical transition phenomena that cannot be attributed to the nonlinear instability.

During the last decade of the 20th century, another viewpoint emerged in the hydrodynamic community on the understanding of the onset of turbulence in spectrally stable shear flows, called bypass transition (cf. Boberg \& Brosa 1988; Butler \& Farrell 1992; Reddy \& Henningson 1993; Trefethen et al. 1993; Morkovin 1993; Gebhardt \& Grossmann 1994; Henningson \& Reddy 1994; Baggett et al. 1995; Waleffe 1997; Grossmann 2000; Reshotko 2001; Chagelishvili et al. 2002; Chapman 2002; Rempfer 2003). Although the bypass transition scenario involves nonlinear interactions - which intervene once the perturbations have reached finite amplitude - the dominant mechanism leading to these large amplitudes appears to be linear. This concept of the onset of turbulence is based on the linear transient growth of vortex mode (aperiodic) perturbations. The potential for transient growth has been recognized for more then a century (see Kelvin 1987; Orr 1907a,b). However, only recently has the importance of the phenomenon been better understood (cf. Moffatt 1967; Marcus \& Press 1977; Gustavsson \& Hultgren 1980; Craik \& Criminale 1986; Farrell \& Ioannou 1993; Reddy \& Henningson 1993; Chagelishvili et al. 1997).

The bypass concept implies that the perturbation energy extracted from the basic flow by linear transient mechanisms causes the increase of the total perturbation energy during the transition process. The nonlinear terms are conservative and only redistribute the energy produced by the linear mechanisms. (A simple sketch of the bypass scenario in a Keplerian flow is given in the Appendix.)

Thus, according to this concept, the transient growth of perturbations (i.e. the linear process) is the key element in the transition to turbulence in spectrally stable flows. The importance of this linear process has been stressed in the titles of several seminal papers: Henningson \& Reddy (1994) ("On the role of linear mechanisms in transition to turbulence”); Baggett et al. (1995) ("A mostly linear model of transition to turbulence") and Reshotko (2001) ("Transient growth: A factor in bypass transition”).

\subsection{On the mechanism of perturbation amplification in spectrally stable shear flows}

The scheme of investigation of shear flow dynamics implies the following steps: introduction of perturbations into a mean flow, linearization of the governing equations, and description of the dynamics of perturbations and flow using the solutions of the initial value problem (i.e. following temporal balances in the flow). In principle this can be done, but in practice it is a formidable task. Therefore, the mathematical approach was changed. This was done by assuming that the solution is separable in eigenmodes, and then establishing the existence of at least one unstable eigensolution. This approach became canonical in time, and resulted in a shift of attention, which became directed to the asymptotic stability of the flow, while no attention was paid to any particular initial value or to the finite time 
period of the dynamics. Indeed, this phase of the evolution was not thought to have any significance - it was left to speculation. But recently the early transient period of the perturbations has been shown to reveal "rich" and complicated behavior leading to different consequences.

It was found in the 1990s that smooth shear flows are crowded by intense processes of mean flow energy extraction by perturbations, energy exchange between perturbations, etc. even in the linear approximation, while following the classical theory they are spectrally stable and consequently "relaxed". It has been especially shown that a superposition of decaying normal modes may grow initially, but will eventually decay as time goes on - a new linear transient channel of energy exchange between the mean flow and perturbations has appeared. Moreover, it has been shown that transient growth can be significant even for subcritical values of the Reynolds number and that its interplay with nonlinear processes can result in the transition to turbulence without any "nonlinear instability" of the flows.

\subsection{On the nonorthogonality of linear operators and the nonmodal approach}

In fact an exact resonance is not necessary to obtain the transient growth of perturbations. This is the consequence of the non-normal character of operators that describe the linear dynamics of perturbations in flows (see, e.g. Reddy et al. 1993). The fact that the eigenfunctions of the linearized Navier-Stokes equations are not orthogonal (i.e. the operator is non-normal) is enough to allow for solutions that exhibit transient growth, depending on the initial conditions, before finally decaying (see Criminale \& Drazin 1990; Trefethen et al. 1993). The mechanism of transient growth is essentially inviscid - the operators are highly non-normal for large Reynolds numbers and the transient growth is asymptotically large in $R e$. (This fact is extremely important in the case of Keplerian accretion disks, where the Reynolds number is literally astronomical: $\operatorname{Re}>10^{10}$ !)

These developments provoked a change of paradigm in the study of linear processes in the considered flows. It was the so-called nonmodal approach that became extensively used and even canonized in the 1990s on these grounds. As a result, substantial progress in the understanding of the shear flow phenomena has been achieved. The nonmodal analysis some modification of the initial value problem - implicates the change of independent variables from a laboratory to a moving frame and the study of temporal evolution of spatial Fourier harmonics (SFH) of perturbations without any spectral expansion in time (see Sect. 4).

\section{The similarity between Keplerian flow and plane parallel shear flow}

Since our aim is to elucidate the basic similarity of the dynamics of plane shear flows and of Keplerian disks, we approximate the Keplerian flow in the two dimensional (2D) limit by its tangent plane parallel flow, while retaining the effect of rotation through the Coriolis force. This model, which ignores the purely geometrical complications, is known as the shearing sheet model; it has been has been used for the study of the linear dynamics of both wave and vortex mode 2D perturbations (cf. Goldreich \& Lynden-Bell 1965; Goldreich \& Tremaine 1978; Drury 1980; Nakagawa \& Sekiya 1992 for wave mode perturbations, and Lominadze et al. 1988; Fridman 1989 and Ioannou \& Kakouris 2001 for vortex mode perturbations).

The dynamical equations are written in the local co-moving Cartesian co-ordinate system:

$x \equiv r-r_{0} ; \quad y \equiv r_{0}\left(\phi-\Omega_{0} t\right)$

where $(r, \phi)$ are standard cylindrical co-ordinates and $\Omega_{0}$ is the local angular velocity at $r=r_{0}$ :

$\Omega(r)=\Omega_{0}+\frac{\partial \Omega}{\partial r}\left(r-r_{0}\right) \equiv \Omega_{0}+A \frac{x}{r_{0}}$

$\left.A \equiv\left(r \partial_{r} \Omega\right)\right|_{r=r_{0}}$ is the shear parameter (in Keplerian disks $A=$ $\left.-3 \Omega_{0} / 2<0\right)$.

For the present purpose, we shall consider only $2 \mathrm{D}$ perturbations, independent of the axial coordinate $z$. The resulting dynamical linear equations for the perturbations of the radial velocity $\left(u_{\mathrm{r}}\right)$, the azimuthal velocity $\left(u_{\phi}\right)$ and the pressure $(p)$ take the form:

$\frac{\partial u_{\mathrm{r}}}{\partial t}+A x \frac{\partial u_{\mathrm{r}}}{\partial y}-2 \Omega_{0} u_{\phi}=-\frac{\partial p}{\partial x}+v \Delta u_{\mathrm{r}}$,

$\frac{\partial u_{\phi}}{\partial t}+A x \frac{\partial u_{\phi}}{\partial y}+2 \Omega_{0} u_{\mathrm{r}}+A u_{\mathrm{r}}=-\frac{\partial p}{\partial y}+v \Delta u_{\phi}$

$\frac{\partial u_{\mathrm{r}}}{\partial x}+\frac{\partial u_{\phi}}{\partial y}=0$

The incompressible limit is taken here to leave out wave mode perturbations and to keep only the vortex mode perturbations, which are the basic ingredient of the bypass scenario. In this case the energy density of the perturbation depends only on its kinematic characteristics and not on the thermodynamics, such as pressure.

Pressure terms are also absent when the dynamical equations are written in vorticity form (for $\omega=$ curl $\mathbf{u}$ ), taking the curl of Eqs. $(3,4)$. This is probably the reason why the pressure perturbations often have been ignored when discussing the dynamics of shear flows. However they play a very important role, even in the incompressible case, because they are the mediators of momentum exchange between fluid particles, which results in the transient growth of the vortex mode. (The physics of the "mediator activity" of the pressure perturbations is described in detail in Chagelishvili et al. 1993, 1996.) The importance of the pressure terms is clearly seen in the following analysis.

We multiply Eqs. (3) and (4) respectively by $u_{\mathrm{r}}$ and $u_{\phi}$, in order to put them in kinetic energy form, and average them over a domain which is symmetrical in $x$. This procedure is similar to that performed in papers by Balbus et al. (1996) and Balbus \& Hawley (1998).

$\frac{\partial}{\partial t}\left\langle\frac{u_{\mathrm{r}}^{2}}{2}\right\rangle=2 \Omega_{0}\left\langle u_{\mathrm{r}} u_{\phi}\right\rangle-\left\langle u_{\mathrm{r}} \frac{\partial p}{\partial x}\right\rangle-v\left\langle\left|\nabla u_{\mathrm{r}}\right|^{2}\right\rangle$, 


$$
\frac{\partial}{\partial t}\left\langle\frac{u_{\phi}^{2}}{2}\right\rangle=-\left[2 \Omega_{0}+A\right]\left\langle u_{\mathrm{r}} u_{\phi}\right\rangle-\left\langle u_{\phi} \frac{\partial p}{\partial y}\right\rangle-v\left\langle\left|\nabla u_{\phi}\right|^{2}\right\rangle .
$$

The source of instability is represented by the shear term $A\left\langle u_{\mathrm{r}} u_{\phi}\right\rangle$. However, the Coriolis term $2 \Omega_{0}\left\langle u_{\mathrm{r}} u_{\phi}\right\rangle$ is explicitly present too, and in Eq. (7) it overbalances the source term, since $A=-3 / 2 \Omega_{0}$ in the Keplerian disk. It thus would seem at first sight that the Coriolis force has a profound influence on the shear flow stability, as has been argued by Balbus \& Hawley (1996), but this conclusion is contradicted by the fact that the Coriolis terms disappear when summing Eqs. (6) and (7), meaning that the growth rate of the total kinetic energy is independent of the Coriolis force.

A more detailed analysis will show that the Coriolis terms may be eliminated also on the level of the dynamical equations, by a suitable renormalization of the pressure perturbation. In this two-dimensional and incompressible case, the perturbation velocity field derives from the stream function $\psi$ :

$u_{\mathrm{r}}=-\frac{\partial \psi}{\partial y} ; \quad u_{\phi}=\frac{\partial \psi}{\partial x}$.

Renormalizing the pressure perturbation

$p^{\mathrm{R}} \equiv p-2 \Omega_{0} \psi$,

Eqs. (3-7) can be rewritten as follows:

$$
\begin{aligned}
& \frac{\partial u_{\mathrm{r}}}{\partial t}+A x \frac{\partial u_{\mathrm{r}}}{\partial y}=-\frac{\partial p^{\mathrm{R}}}{\partial x}+v \Delta v_{r}, \\
& \frac{\partial u_{\phi}}{\partial t}+A x \frac{\partial u_{\phi}}{\partial y}+A u_{\mathrm{r}}=-\frac{\partial p^{\mathrm{R}}}{\partial y}+v \Delta v_{\phi},
\end{aligned}
$$

$\frac{\partial u_{\mathrm{r}}}{\partial x}+\frac{\partial u_{\phi}}{\partial y}=0$

$\frac{\partial}{\partial t}\left\langle\frac{u_{\mathrm{r}}^{2}}{2}\right\rangle=-\left\langle u_{\mathrm{r}} \frac{\partial p^{\mathrm{R}}}{\partial x}\right\rangle-v\left\langle\left|\nabla u_{\mathrm{r}}\right|^{2}\right\rangle$

$\frac{\partial}{\partial t}\left\langle\frac{u_{\phi}^{2}}{2}\right\rangle=-A\left\langle u_{\mathrm{r}} u_{\phi}\right\rangle-\left\langle u_{\phi} \frac{\partial p^{\mathrm{R}}}{\partial y}\right\rangle-v\left\langle\left|\nabla u_{\phi}\right|^{2}\right\rangle$.

In Sect. 2 we outlined the bypass concept in Cartesian/planar shear flows (a simple sketch of the bypass scenario is presented in the Appendix). Since we wish to apply a similar scenario to differentially rotating disks, we shall write the Cartesian counterparts of the averaged equations for comparison. If $y$ is the streamwise variable, and $x$ is the shearwise variable as in the considered case (i.e., $x$ is analog to $r$, and $y$ to $\phi$ ), then the equations are:

$$
\begin{aligned}
& \frac{\partial}{\partial t}\left\langle\frac{u_{x}^{2}}{2}\right\rangle=-\left\langle u_{x} \frac{\partial p^{\mathrm{c}}}{\partial x}\right\rangle-v\left\langle\left|\nabla u_{x}\right|^{2}\right\rangle, \\
& \frac{\partial}{\partial t}\left\langle\frac{u_{y}^{2}}{2}\right\rangle=-A^{\mathrm{c}}\left\langle u_{x} u_{y}\right\rangle-\left\langle u_{y} \frac{\partial p^{\mathrm{c}}}{\partial y}\right\rangle-v\left\langle\left|\nabla u_{y}\right|^{2}\right\rangle,
\end{aligned}
$$

where $A^{\mathrm{c}}$ is the shear parameter of the Cartesian flow $U_{0}^{\mathrm{c}}\left(0, A^{\mathrm{c}} x\right) ; \quad u_{x}, \quad u_{y}$ and $p^{\mathrm{c}}$ are the perturbations of velocity and pressure, respectively. If the flow is set up with $U_{0}^{\mathrm{c}}$ decreasing with $x$ (i.e. $A^{\mathrm{c}}<0$ ), then Eq. (16) shows that steady flow is marked by transport in the direction of increasing $x$, as in the Keplerian disk.

These equations are identical to the normalized equations derived above for the Keplerian case, which stresses the similarity of these two flows. But they deal with spatially averaged quantities, and one may wish to establish that the similarity is even more profound, namely that the local kinematics and energy of 2D vortex perturbations of plane and disk flows are the same in time and space, starting from identical initial conditions.

\section{Transient growth in 2D}

Mainly for the purpose of illustrating the bypass mechanism, we shall describe now the evolution in time of an initial perturbation, in two dimensions. We perform a spatial Fourier transform of all relevant variables, as shown here for the pressure fluctuation:

$p(x, y, t)=\hat{p}\left(k_{x}(t), k_{y}, t\right) \exp \left[\mathrm{i} k_{x}(t) x+\mathrm{i} k_{y} y\right]$.

In a shearing flow, the perturbations cannot keep the form of a simple wave, since the wave-number of each spatial Fourier harmonics (SFH) depends on time (see Criminale \& Drazin 1990 for a rigorous mathematical interpretation). In our geometry

$k_{x}(t)=k_{x}(0)-A k_{y} t$,

thus the wave-number of each SFH varies in time along the flow shear: it "drifts" in k-space. Therefore $p$, in Eq. (17) above, depends on time through $k_{x}(t)$.

Equations (5), (6) and (7) take the following form in Fourier space:

$$
\begin{aligned}
\frac{\mathrm{d}}{\mathrm{d} t} \hat{u}_{\mathrm{r}}\left(k_{x}(t), k_{y}, t\right)- & 2 \Omega \hat{u}_{\phi}\left(k_{x}(t), k_{y}, t\right) \\
= & -\mathrm{i} k_{x}(t) \hat{p}\left(k_{x}(t), k_{y}, t\right),
\end{aligned}
$$

$$
\begin{array}{r}
\frac{\mathrm{d}}{\mathrm{d} t} \hat{u}_{\phi}\left(k_{x}(t), k_{y}, t\right)+(2 \Omega+A) \hat{u}_{\mathrm{r}}\left(k_{x}(t), k_{y}, t\right) \\
=-\mathrm{i} k_{y} \hat{p}\left(k_{x}(t), k_{y}, t\right), \\
k_{x}(t) \hat{u}_{\mathrm{r}}\left(k_{x}(t), k_{y}, t\right)+k_{y} \hat{u}_{\phi}\left(k_{x}(t), k_{y}, t\right)=0 .
\end{array}
$$

For simplicity, we ignored here the viscous forces; their action may be easily incorporated in the analysis, as we shall see below.

One readily shows that these equations possess a time invariant

$\mathcal{I}=k_{y} \hat{u}_{\mathrm{r}}(t)-k_{x}(t) \hat{u}_{\phi}(t)$

which expresses the conservation of vorticity in Fourier space. Making use of this invariant, we may write the solution of the system (19-21) as follows:

$\hat{u}_{\mathrm{r}}\left(k_{x}(t), k_{y}, t\right)=\frac{k_{y}}{k_{x}^{2}(t)+k_{y}^{2}} \mathcal{I}$, 


$$
\begin{aligned}
& \hat{u}_{\phi}\left(k_{x}(t), k_{y}, t\right)=-\frac{k_{x}(t)}{k_{x}^{2}(t)+k_{y}^{2}} \mathcal{I}, \\
& \hat{p}\left(k_{x}(t), k_{y}, t\right)=\frac{\mathrm{i}\left[A k_{y}^{2}+2 \Omega_{0}\left(k_{x}^{2}(t)+k_{y}^{2}\right)\right]}{\left[k_{x}^{2}(t)+k_{y}^{2}\right]^{2}} \mathcal{I} .
\end{aligned}
$$

We see that the perturbed quantities vary aperiodically in time (as is natural of vortex mode perturbations), and that they undergo transient amplification. Energy is exchanged between the perturbations and the background flow, and that represents the basis of the bypass transition scenario. It is important to note that the kinematic characteristics of the perturbations do not depend on the rotation rate $\Omega_{0}$ - they are identical to those of Cartesian shear flows. Only the pressure perturbation depends on $\Omega_{0}$. Consequently, the energetics of the SFH is identical to that of the Cartesian case:

$$
\begin{aligned}
\hat{u}^{2}\left(k_{x}(t), k_{y}, t\right) & \equiv \hat{u}_{\mathrm{r}}^{2}\left(k_{x}(t), k_{y}, t\right)+\hat{u}_{\phi}^{2}\left(k_{x}(t), k_{y}, t\right)=\frac{1}{k_{x}^{2}(t)+k_{y}^{2}} \mathcal{I}^{2} \\
& =\hat{u}^{2}\left(k_{x}(0), k_{y}, 0\right) \frac{k_{x}^{2}(0)+k_{y}^{2}}{k_{x}^{2}(t)+k_{y}^{2}} .
\end{aligned}
$$

We see that the kinetic energy reaches maximum amplitude for $k_{x}(t)=0$. Similarly, the renormalized pressure perturbations do not involve $\Omega_{0}$ :

$$
\begin{aligned}
\hat{p}^{\mathrm{R}}\left(k_{x}(t), k_{y}, t\right) & =\hat{p}\left(k_{x}(t), k_{y}, t\right)-2 \Omega_{0} \hat{\psi}\left(k_{x}(t), k_{y}, t\right) \\
& =\frac{\mathrm{i} A k_{y}^{2}}{\left[k_{x}^{2}(t)+k_{y}^{2}\right]^{2}} \mathcal{I} .
\end{aligned}
$$

This expression is identical to that describing the evolution of the pressure perturbation in a Cartesian flow, which proves the similarity of transient growth in both types of flows, in two dimensions.

The above analysis has been done in the inviscid case. Accounting for the viscous forces is a straightforward procedure (see Fridman 1989): it may be done by multiplying the obtained equations by the factor $\exp \left[-v \int_{0}^{t} \mathrm{~d} t^{\prime}\left(k_{x}^{2}\left(t^{\prime}\right)+k_{y}^{2}\right)\right]$. For instance, Eqs. (19) and (26) will read as follows:

$$
\begin{aligned}
& \hat{u}_{\mathrm{r}}\left(k_{x}(t), k_{y}, t\right)=\frac{k_{y} \mathcal{I}}{k_{x}^{2}(t)+k_{y}^{2}} \mathrm{e}^{-v} \int_{0}^{t} \mathrm{~d} t^{\prime}\left[k_{x}^{2}\left(t^{\prime}\right)+k_{y}^{2}\right] \\
& \hat{u}^{2}\left(k_{x}(t), k_{y}, t\right)=\frac{\mathcal{I}^{2}}{k_{x}^{2}(t)+k_{y}^{2}} \mathrm{e}^{-2 v \int_{0}^{t} \mathrm{~d} t^{\prime}\left[k_{x}^{2}\left(t^{\prime}\right)+k_{y}^{2}\right]} .
\end{aligned}
$$

To estimate the maximum amplification which is achieved in this transient growth, let us express $k_{x}^{2}(0) / k_{y}^{2}$ in terms of the Reynolds number that we define as $R e=\Omega_{0} r_{0}^{2} / v$. The value $k_{x}^{2}(0)$ may be deduced from the local balance between transient growth and viscous dissipation for this smallest scale: $v k^{2} \hat{u}^{2}=\left|A \hat{u}_{x} \hat{u}_{y}\right|=\left|A k_{x}(0) k_{y} / k^{2}\right| \hat{u}^{2}$ (see Chagelishvili et al. 2002). Taking into account that $k^{2}=k_{x}^{2}(0)+k_{y}^{2} \approx k_{x}^{2}(0)$ and combining the above relations one gets:

$\frac{\hat{u}_{\max }^{2}}{\hat{u}^{2}\left(k_{x}(0), k_{y}, 0\right)} \approx \frac{k_{x}^{2}(0)}{k_{y}^{2}} \approx \frac{2}{3}\left(\frac{R e}{\left(k_{y} r_{0}\right)^{2}}\right)^{2 / 3}$,

which can reach a huge value in astrophysical disks.

\section{Discussion and conclusions}

We have seen that the concept of nonlinear instability in smooth spectrally stable shear flows has undergone substantial revision in the hydrodynamics community. Nowadays the concept of the bypass transition to turbulence has become a favorite and is under intensive development.

Let us summarize the main features of this concept:

- The onset of turbulence and the turbulent state itself in smooth spectrally stable shear flows is supported energetically by the linear transient growth of vortex mode perturbations the key ingredient of the turbulence is the vortex mode (eddy) perturbation, and the key phenomenon is the linear transient growth of the perturbation.

- Nonlinear processes do not contribute to any energy growth, but regenerate vortex mode perturbations that are able to extract shear flow energy. Doing so, nonlinear processes only indirectly favor the energy extraction by the vortex mode perturbations.

- The non-orthogonal nature of the linearized Navier-Stokes equations is the formal basis of the transient growth.

- The non-orthogonal nature increases with increasing Reynolds number; thus the operators are highly non-normal for the huge Reynolds numbers of Keplerian disks $\left(\operatorname{Re}>10^{10}\right)$ and the transient growth is asymptotically large in $R e$.

In this paper we wanted to contribute to the revival of hydrodynamic shear turbulence as a possible explanation for the "anomalous" viscosity in non-magnetized Keplerian accretion disks. Specifically, we wished to draw the attention of astrophysicists to the bypass concept.

We showed that in disk flows there exist vortex mode perturbations which are similar to those that are held responsible for the onset of turbulence in the Cartesian shear flow. The key point of our analysis is the interpretation of the important role of the pressure perturbations in the dynamical processes.

In fact, the kinematics and energetics of the vortex mode perturbations are identical in the rotating disk and the Cartesian shear flows in the 2D case. The Coriolis force only causes deviation of the pressure perturbations from the Cartesian case. By focusing on the epicyclic motions, and underestimating the action of the pressure terms, one is led to the false conclusion that the Coriolis force suppresses hydrodynamic turbulence in Keplerian flows (Balbus et al. 1996; Balbus \& Hawley 1998).

This property of the pressure perturbations has been established here in the $2 \mathrm{D}$ case. In the more general case of $3 \mathrm{D}$ perturbations, the dynamics of vortex mode perturbations is somewhat more complicated, as it will be shown in a forthcoming paper (Tevzadze et al.). However, the role of the pressure perturbations is still to counteract the Coriolis force. In the 3D disk case, the wave-number domain where the perturbation undergoes transient growth is smaller in comparison to 3D Cartesian flows, but this is compensated for by the very large Reynolds number characterizing astrophysical disks.

Other scenarios which may lead to hydrodynamic turbulence in astrophysical disks have been presented recently: they invoke a linear (spectral) instability arising from the stratification perpendicular to the disk (Dubrulle et al. 2002) or due to deviations from cylindrical rotation (Urpin 2002; 
Klahr \& Bodenheimer 2002). Such instabilities may well compete with the bypass mechanism presented here, and it is not possible to conclude presently which is the best candidate to render astrophysical disks turbulent.

In laboratory experiments the field narrows, because there is no stratification in the fluid (other than imposed on purpose), and there the bypass mechanism provides an attractive explanation for the turbulence detected in flows that are linearly stable (angular momentum increasing outwards). We refer to CouetteTaylor experiments performed by Wendt (1933), Taylor (1936), Coles (1950) and Van Atta (1966). Very recently such turbulence has been observed also in rotation profiles which share the properties of Keplerian disks, namely with their angular velocity decreasing outwards (Richard 2001).

Decisive conclusions about the self-sustenance of the turbulent state need to be supported by numerical simulations. To our knowledge, the simulations reported so far have failed to detect hydrodynamic shear turbulence in rotating flows with angular momentum increasing outwards. In a recent paper, Longaretti (2002) has discussed the possible explanations for this negative result. The main reason is probably the lack of spatial resolution, which still prevents us from reaching even the Reynolds numbers at which turbulence is detected in the laboratory. At this stage, we hope that the analysis presented here will help to build a fruitful background for the nonlinear numerical stability analysis of rotating astrophysical shear flows. A definite answer will result from the convergence of theoretical, numerical and experimental work.

Acknowledgements. This work is supported by the International Science and Technology Center grant G-553. G.D.C. would like to acknowledge the hospitality of Observatoire de Paris (LUTH). The authors would like to thank the referee for helpful comments on the early version of the paper.

\section{Appendix A: Bypass scenario of the transition to turbulence}

We present here a simple sketch of the bypass scenario applied to Keplerian disk flow, in the wave-number plane $\left(k_{x}, k_{y}\right)$ (see Fig. A.1). We shall define as the "active domain" the region where viscous dissipation may be neglected, i.e. where $k_{x}^{2}+k_{y}^{2}<k_{v}^{2}$, with $k_{v} \approx R e^{3 / 4} / r_{0}$.

The linear dynamics of the perturbation may be described by following each of its spatial Fourier harmonics (SFH) in this wave-number plane. We single out one that is located initially at some point 1 in the amplification part of the "active domain", which meets the condition $k_{x} / k_{y}<0$. According to Eq. (18), as $k_{x}(t)$ varies in time, the SFH drifts in the direction marked by the arrows, since $A<0$. (We present the drift of the SFH only in the upper half-plane; since the perturbation is real, there is a counterpart in the lower half-plane.) Initially, as $\left|k_{x}(t)\right|$ decreases, the energy of the SFH grows. This growth lasts until the wave vector crosses the line $k_{x}=0$ (point 2). Then, while undergoing attenuation, the SFH continues its drift until it reaches point 3 , where it is dissipated through viscous friction. The same will occur with all other Fourier harmonics. Consequently, if the nonlinear interaction between different Fourier harmonics is inefficient, the perturbation disappears

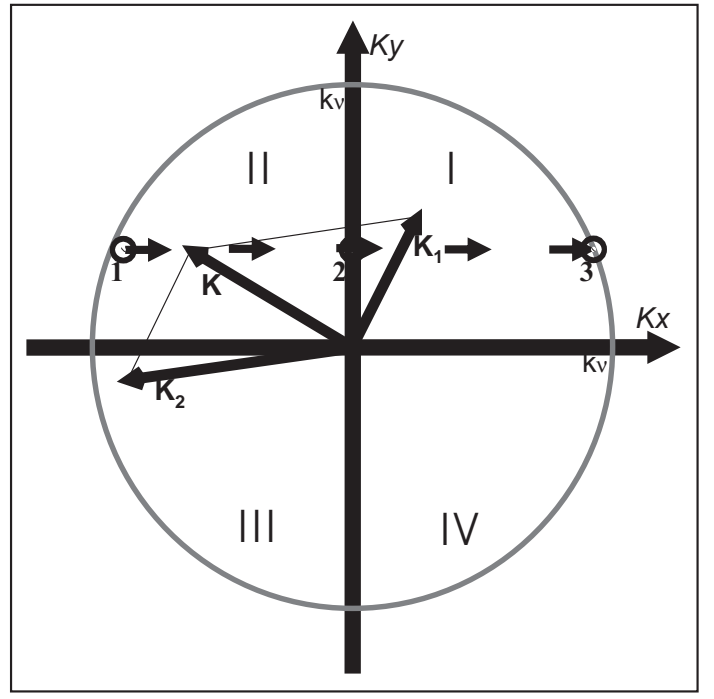

Fig. A.1. A simple sketch of the bypass scenario applied to Keplerian disk flow, in the wave-number plane $\left(k_{x}, k_{y}\right) . k_{x}$ is the wave-number in the radial direction and $k_{y}$ in the azimuthal direction. The wavenumber of the spatial Fourier harmonics (SPH) drifts from its initial position 1, the SPH is amplified in quadrant II, reaches maximum amplitude in 2, is attenuated in quadrant I, and undergoes viscous dissipation in 3. But the amplification quadrant II is repopulated through nonlinear interaction from SFHs located in the attenuation quadrants I and III. (See text for more details.)

eventually. Permanent extraction of shear energy by the perturbations is necessary for their maintenance, which is possible when quadrants II and IV where $k_{x} / k_{y}<0$ are being repopulated through nonlinear interactions between Fourier harmonics of quadrants I and III that have reached sufficient amplitude. This may be achieved through three-wave processes $\mathbf{k}_{\mathbf{1}}+\mathbf{k}_{\mathbf{2}} \Rightarrow \mathbf{k}$; four wave processes $\mathbf{k}_{\mathbf{1}}+\mathbf{k}_{\mathbf{2}}+\mathbf{k}_{\mathbf{3}} \Rightarrow \mathbf{k}$; five wave processes, etc. In the figure we present an example of three wave process $\mathbf{k}_{\mathbf{1}}+\mathbf{k}_{\mathbf{2}} \Rightarrow \mathbf{k}$ that contributes to the regeneration of an SFH in the amplification area, transferring perturbation energy to it from the attenuation areas. The bypass scenario implies the dominance of this regeneration tendency of nonlinear processes, i.e. predominant transfer of perturbation energy from quadrants I and III to quadrants II and IV, in other words nonlinear positive feedback. To what extent the reproduction of the Fourier harmonics in quadrants II and IV is sustained, even in the case of positive feedback, depends both on the amplitude and on the spectrum of the initial perturbation. Nonlinear decay processes are weak at low amplitudes and are not able to compensate the linear drift of SFH in k-plane. As a result, weak perturbations are damped without any trace, and without inducing transition to turbulence. The higher the amplitude of initial perturbation the stronger are the nonlinear effects. At a certain amplitude (which, of course, depends on the initial vortex perturbation spectrum in $\mathbf{k}$-plane and on the Reynolds number), the nonlinear processes are able to counterbalance the action of the linear drift, thus ensuring permanent return of SFH to the amplification areas (quadrants II and IV). This eventually ensures a permanent extraction of energy from the background flow and maintenance of the perturbations, and thus of turbulence. 
Therefore the bypass scenario can be realized only in the case of finite amplitude perturbations and in each case it has a threshold that depends on the perturbation spectrum and the Reynolds number.

Let us summarize. According to the bypass concept, vortex mode (eddy) perturbations are the basic ingredient of hydrodynamic shear turbulence. The bypass scenario involves the interplay of four basic phenomena:

- the linear "drift" of SFH in the k-plane;

- the transient growth of SFH;

- the usual viscous dissipation;

- nonlinear processes that close the feedback loop of the transition by mixing - by the angular redistribution of SFH in k-plane.

\section{References}

Baggett, J. S., Driscoll, T. A., \& Trefethen, L. N. 1995, Phys. Fluids, 7,833

Batler, K. M., \& Farrel, B. F. 1992, Phys. Rev A, 4, 1637

Balbus, S. A., \& Hawley, J. F. 1991, ApJ, 376, 214

Balbus, S. A., \& Hawley, J. F. 1992, ApJ, 400, 610

Balbus, S. A., Hawley, J. F., \& Stone, J. M. 1996, ApJ, 467, 76

Balbus, S. A., \& Hawley, J. F. 1998, Rev. Mod. Phys., 70, 1

Bayly, B. J. 1986, Phys. Rev. Lett., 57, 2160

Bayly, B. J., Orszag, S. A., \& Herbert, T. 1988, Ann. Rev. Fluid Mech., 20,359

Broberg, L., \& Brosa, U. 1988, Z. Naturforschung Teil, 43a, 697

Chagelishvili, G. D., Khristov, T. S., Chanishvili, R. G., \& Lominadze, J. G. 1993, Phys. Rev. E, 47, 366

Chagelishvili, G. D., Chanishvili, R. G., \& Lominadze, J. G. 1996, JETP Lett., 63, 543

Chagelishvili, G. D., Tevzadze, A. G., Bodo, G., \& Moiseev, S. S. 1997, Phys. Rev. Lett., 79, 3178

Chagelishvili, G. D., Chanishvili, R. G., Hristov, T. S., \& Lominadze, J. G. 2002, JETP, 94, 434

Chapman, S. J. 2002, J. Fluid Mech., 451, 35

Coles, D. 1965, J. Fluid Mech., 21, 385

Craik, A. D. D., \& Criminale, W. O. 1986, Pros. R. Soc. Lond. A, 406, 13

Criminale, W. O., \& Drazin, P. G. 1990, Studies Appl. Math., 83, 123

Drury, L. O'C. 1980, MNRAS, 193, 337

Dubrulle, B., Marié, L., Normand, Ch., \& Zahn, J.-P. et al. 2002, A\&A, submitted

Farrell, B. F., \& Ioanou, P. J. 1993, Phys. Fluids A, 5, 1390

Fjørtøft, R. 1950, Geofis. Publ., 17, 52

Fridman, A. M. 1989, Sov. Astr. Lett., 15, 487

Gebhardt, T., \& Grossmann, S. 1994, Phys. Rev. E, 50, 3705
Goldreich, P., \& Lynden-Bell, D. 1965, MNRAS, 130, 125

Goldreich, P., \& Tremaine, S. D. 1978, ApJ, 222, 850

Grossmann, S. 2000, Rev. Mod. Phys., 72, 603

Gustavsson, L. H., \& Hultgren, L. S. 1980, J. Fluid Mech., 98, 149

Hawley, J. F., \& Balbus, S. A. 1991, ApJ, 376, 223

Hawley, J. F., \& Balbus, S. A. 1992, ApJ, 400, 595

Hawley, J. F., Gammie, C. F., \& Balbus, S. A. 1995, ApJ, 440, 742

Henningson, D. S., \& Reddy, S. C. 1994, Phys. Fluids, 6, 1396

Ioannou, P. J., \& Kakouris, A. 2001, ApJ, 550, 931

Klahr, H. H., \& Bodenheimer, P. 2002 [astro-ph/0211629]

Longaretti, P.-Y. 2002, ApJ, 576, 587

Lominadze, J. G., Chagelishvili, G. D., \& Chanishvili, R. G. 1988, Sov. Astr. Lett., 14, 364

Lord Kelvin 1887, Philos. Mag., 23, 459

Lynden-Bell, D. 1969, Nature, 223, 690

Marcus, P., \& Press, W. H. 1977, J. Fluid Mech., 79, 525

Moffatt, K. 1967, in Atmospheric Turbulence and Radio Wave Propagation, ed. A. M. Yaglom, \& V. I. Tatarskii (Moscow: Nauka Press)

Morkovin, M. V. 1993, in Instabilities and Turbulence in Engineering flows, ed. D. E. Ashpis, T. B. Gatski, \& R. S. Hirsh (New York: Kluwer Academic Press)

Nakagawa, Y., \& Sekiya, M. 1992, MNRAS, 256, 685

Orr, W. McF. 1907a, Proc. R. Irish Acad. Sci. A, 27, 9

Orr, W. McF. 1907b, Proc. R. Irish Acad. Sci. A, 27, 69

Orszag, S. A., \& Kells, L. C. 1980, J. Fluid Mech., 96, 161

Orszag, S. A., \& Patera, A. T. 1980, Phys. Rev. Lett., 45, 989

Orszag, S. A., \& Patera, A. T. 1983, J. Fluid Mech., 128, 347

Pringle, J. E. 1981, ARA\&A, 19, 137

Rayleigh, Lord 1880, Scientific Papers, 1, 474 (Cambridge Univ. press)

Reddy, S. C., \& Henningson, D. S. 1993, J. Fluid Mech., 252, 209

Reddy, S. C., Schmid, P. J., \& Hennigson, D. S. 1993, SIAM J. Appl. Math., 53, 15

Rempfer, D. 2003, Annu. Rev. Fluid Mech., 35, 229

Reshotko, E. 2001, Phys. Fluids, 13, 1067

Richard, D. 2001, Thèse de doctorat, Université Paris 7

Richard, D., \& Zahn, J.-P. 1999, A\&A, 347, 734

Shakura, N. I., \& Sunyaev, R. A. 1973, A\&A, 24, 337

Stone, J. M., Hawley J. F., Gammie, C. F., \& Balbus, S. A. 1996, ApJ, 463,656

Taylor, G. I. 1936, Proc. R. Soc. Lond. A, 157, 546

Tevzadze, A. G., Chagelishvili, G. D., Chanishvili, R. G., Lominadze, J. G., \& Zahn, J.-P., in preparation

Trefethen, L. N., Trefethen, A. E., Reddy, S. C., \& Discoll, T. A. 1993 , Science, 261, 578

Urpin, V. 2002, A\&A, submitted

Van Atta, C. W. 1966, J. Fluid Mech., 25, 495

Waleffe, F. 1997, Phys. Fluids, 9, 883

Wendt, F. 1933, Ing. Arch., 4, 577 